\title{
Effectiveness of Exercise Regimen on Quality of Sleep in Patients with End Stage Renal Disease on Maintenance Haemodialysis
}

\author{
Nishtha Mehta ${ }^{1}$, Meena Kumari ${ }^{2}$, Rubal $^{3}$, Sakshee Sharma ${ }^{4}$, Sudhir Kumar ${ }^{5}$ \\ ${ }^{1}$ Nursing Tutor, Chitkara School of Health Sciences, Chitkara University, Punjab, India, ${ }^{2}$ Staff Nurse, Neuro \\ Science deparment, Max Superspeciality Hospital, Patparganj, Delhi, India, ${ }^{3}$ Staff Nurse, Abhilasha Hospital, \\ Chandigarh, India, ${ }^{4}$ Clinical instructor, Chitkara School of Health Sciences, Chitkara University, Punjab, India, \\ ${ }^{5}$ Quality Regional Manager, Quantzo Health Services, New Delhi, India
}

\begin{abstract}
Background: Patients on maintenance haemodialysis suffering from sleep problem, Exercise makes the patient tired and improves the circulation in the body which leads to induction of sleep. The present study aimed to evaluate the effectiveness of exercise regimen on quality of sleep of patients.
\end{abstract}

Materials and Method: A pre-experimental research design was used to assess quality of sleep among 30 patients with End Stage Renal Disease on Maintenance Haemodialysis. P atients were allocated to intervention group via purposive sampling technique. Intervention for intervention group included exercise for two weeks ( 5 days in a week). Interview based standardized questionnaire was used to assess the quality of sleep by using Pittsburgh Sleep Quality Index (PSQI) in associated with clinical and lab values .Patients were followed on every day for two weeks by using phone call reliability and feedback form.

Result: Pre test sleep quality score was $(9.766 \pm 1.887)$ and post- intervention sleep quality scorewas $(7.60 \pm$ 2.175), there is a decrease in score of sleep; hence there is positive effect of exercise regimen on sleep. Labvalues: calcium, platelets, chloride and albumin are having significant relation with sleep quality by using regression analysis.

Conclusion: Non pharmacological method are more effective to improve the quality of sleep among patient on maintenance haemodialysis having sleep problem.

Keywords: Haemodialysis, Sleep quality, Pittsburgh sleep Quality (PSQI), Ca, platelet, Chloride, Albumin.

\section{Introduction}

Sleep is a common problem among patients with end stage renal disease on maintenance hemodialysis due to excessive accumulation of fluids in the body. Most of the reported complaints are insomnia, restless leg syndrome

\section{Corresponding Author:}

\section{Ms. Nishtha Mehta}

Nursing Tutor, Chitkara School of Health Sciences, Chitkara University, Punjab, India

e-mail: nishtha@chitkara.edu.in

Mobile No.: 7889192301
(RLS), sleep-disordered breathing and excessive daytime sleepiness (EDS). ${ }^{1}$ It has been reported that $80 \%$ of ESRD patients receiving dialysis reports sleep complaints with daytime sleepiness. ${ }^{2}$ In healthy individual .sleep is lead by decrease in sympathetic activity and increase in vagal tone that leads to reduction of blood pressure at night. ${ }^{3}$. According to Hilderth Most of the Patients with ESRD are having sympathethic-vagal imbalance due to baro receptor reflex malfunctioning in which there is a hyperactivity of the sympathetic nervous system and decreased vagal tone. ${ }^{4}$

As for the sleep - wake circadian rhythm melatonin hormone is responsible. It is secreted in less amount 
during the daytime but increases its amount during the night, which helps in falling asleep. But in ESRD patients the amount of melatoninsecreted by pineal gland is less which affects the sleep cycle. ${ }^{5}$

Also there is overnight rostral shifting of fluid from legs toward neck and result in restriction and collapse of upper airway. Pathogenesis of Obstructivesleep apnea is unknown but Rosilene $\mathbf{M}$, hypothesed that changes in neck circumference and severity of obstructive sleep apnea is related to overnight shifting of leg fluid volume in ESRD patients. ${ }^{6}$ Physical activity improves sleep quality and increase sleep duration. Exercises increase the circulation of blood in the body. Exercise makes the patient tired and reduces the stress, which help in induction of sleep. Hence simple to moderate exercise results in maintenance of good sleep quality among hemodialysis patients. The workout about 30 minutes in a day 5 times a week,if cant fit it even just having 10 minutes a day of walking,swimming or riding can help in getting night sleep("National sleep foundation") ${ }^{7}$

\section{Materials and Method}

A pre- experimental research design was conducted in dialysis unit of Fortis Hospital, Mohali. The permission taken from the Research and ethical committee of the Hospital. Written informed consent form were filled from the patients suffering from sleep problem.

Inclusion criteria of the study was Patients with End Stage Renal Disease, Above 18 years of age, Consenting for participation in study, who were able to walk, Patient able to carry out intervention and Patients who were willing to participate in the study. Exclusion criteria of the study was Patients with-Any other co-morbidity like liver disease, infected patients/HIV,Patients admitted in intensive care units, Any recent major post-operative patients, History of any analgesics medication (opiod and non opoid)and Patients going for evening walk as normal routine activity.
After fulfilling the inclusion and exclusion criteria,Sociodemograohic data collected.Patients Lab Values :RFT's,LFT's and Urine test sample taken .Patient comorbities data also collected .

Exercise intervention was provided to the patients. Patients were asked to do evening walk of (25-30 minutes) for consecutive two weeks (five days in a week). Phone call based reliability of exercise was done on daily basis from the day of starting till the end of intervention i.e., evening walk.Written feedback was taken in mid of the intervention period by using feedback form for assessing any difficulties, confusions and their experience during the walk. Subjects were evaluated on eleventh day of intervention period to obtain posttest information regarding clinical variables and sleep quality by using Pittsburgh Sleep Quality Index. ${ }^{8}$

\section{Findings:}

Table 1: Comparison of level of sleep quality among haemodialysis patients with End stage Renal Disease. $\mathbf{N}=\mathbf{3 0}$

\begin{tabular}{|l|c|c|}
\hline $\begin{array}{l}\text { Level of sleep } \\
\text { quality score }\end{array}$ & Pre test f(\%) & Post test f (\%) \\
\hline Very good $(0)$ & $00(0 \%)$ & $00(0 \%)$ \\
\hline Fairly good (1-7) & $2(6.7 \%)$ & $13(43.3 \%)$ \\
\hline Fairly bad (8-14) & $27(90.0 \%)$ & $16(53.3 \%)$ \\
\hline Very bad (15-21) & $01(3.3 \%)$ & $01(3.3 \%)$ \\
\hline
\end{tabular}

No difficulty: - 0 Severe difficulty: - 21

Table No.1 depicts the comparison of level of pretest and posttest scores of sleep quality. It shows significant improvement from pretest to posttest scores i.e., there is decrease in percentage of fairly bad sleep lie from $90 \%$ to $53.3 \%$ and increase in percentage of fairly good sleep lie from $6.7 \%$ to $43.3 \%$

\section{Table 2: Comparison of mean pretest and mean post test score of sleep quality among haemodialysis patients with End Stage Renal Diseases. $\mathbf{N}=\mathbf{3 0}$}

\begin{tabular}{|c|c|c|c|c|c|c|}
\hline \multirow{2}{*}{ Subject with Sleep Quality } & \multicolumn{2}{|c|}{ Mean sleep quality score } & \multirow{2}{*}{$\begin{array}{c}\text { Mean } \\
\text { difference }\end{array}$} & \multirow{2}{*}{$\begin{array}{l}\text { Standard } \\
\text { Deviation }\end{array}$} & \multirow{2}{*}{ t-value } & \multirow{2}{*}{ p value } \\
\hline & Pretest & Posttest & & & & \\
\hline Experimental Group $\mathrm{N}=30$ & 9.766 & 7.60 & 0.367 & 0.490 & 4.097 & $0.001 *$ \\
\hline
\end{tabular}

Level of significance $=0.05 *$ Significant $=p<0.05$

Minimum $=0$ Maximum $=21$ 
Table No 2 depicts the comparison of mean pretest and mean posttest scores of sleep quality among hemodialysis patients. Paired T-test used for the comparison which shows mean, mean difference, standard deviation $\mathrm{t}$ - value and $\mathrm{p}$ value. It shows significant result as $\mathrm{p}$ value is $<0.005$. $\mathrm{P}$ - Value is 0.001 , it shows that our null hypothesis was rejected and research hypothesis was accepted i.e., "There is significant effect of exercise on sleep quality in patients with End Stage Renal Disease on Maintenance Haemodialysis".

Table 3: Comparison of various components of sleep quality among patients on hemodialysis. $\mathbf{N}=\mathbf{3 0}$

\begin{tabular}{|l|c|c|c|c|}
\hline Components of sleep quality & Pretest Mean + S.D. & Posttest Mean + S.D. & t value & p value \\
\hline Subjective Sleep quality & $1.53+0.571$ & $1.13+0.346$ & 4.397 & $0.001^{* *}$ \\
\hline Sleep Latency & $2.17+0.648$ & $1.73+0.691$ & 4.176 & $0.001^{* *}$ \\
\hline Sleep duration & $1.83+0.592$ & $1.33+0.547$ & 4.785 & $0.001^{* *}$ \\
\hline Habitual sleep efficiency & $1.40+0.932$ & $0.97+0.928$ & 2.282 & $0.030^{*}$ \\
\hline Sleep disturbances & $1.37+0.490$ & $1.13+0.346$ & 2.971 & $0.006^{* *}$ \\
\hline Use of sleeping medication & - & - & - & 1.651 \\
\hline Daytime functions & $1.50+0.731$ & $1.27+0.640$ & & 0.109 \\
\hline
\end{tabular}

Level of significance $=0.05 *$ Significant $=\mathrm{p}<0.05$

Table 3 :Depicts the comparison of various seven components of sleep quality. Paired T- test was used for comparison which shows mean with standard deviation, $t$ value and $p$ value of all the components. All the components were significant because there $p$ value was
$<0.005$ and daytime functions was not significant as its $\mathrm{p}$ value was $>0.05$.

As sleep medication was our exclusion criteria, no patient were chosen who are on sleep medication

Table No. 4: Association of clinical variables with sleep quality.

\begin{tabular}{|l|c|c|c|c|}
\hline Clinical Variables & Unstandardized B Coefficients & Standardized B Coefficients & t- value & p- value \\
\hline Calcium $(\mathrm{mg} / \mathrm{dl})$ & 0.836 & 0.496 & 3.663 & $0.001 * *$ \\
\hline Platelets $($ thou/ul) & 0.011 & 0.457 & -2.519 & $0.0002 * *$ \\
\hline Chloride $(\mathrm{mEq} / \mathrm{L})$ & -0.157 & -0.335 & -2.145 & $0.019 *$ \\
\hline Albumin $(\mathrm{gm} / \mathrm{dl})$ & -0.417 & -0.289 & $0.042^{*}$ \\
\hline
\end{tabular}

Table 4 depicts the association of clinical variables with the sleep quality. It shows the $B$ coefficients, $t$ value and $\mathrm{p}$ value.

A stepwise regression was performed, four variables were included in the final model such as calcium $(\mathrm{B}=$ 0.836 , $p$ value $=0.001)$, followed by platelets $(B=0.011$, $\mathrm{p}$ value $=0.0002)$, chloride $(\mathrm{B}=-0.157, \mathrm{p}$ value $=0.0 \mathrm{~s} 19)$ and $\operatorname{albumin}(\mathrm{B}=-0.417, \mathrm{p}$ value $=0.042)$.

On the basis of standardized B coefficients the order of influence on sleep quality was calcium $(B=0.496)$, followed by platelets $(\mathrm{B}=0.457)$, chloride $(\mathrm{B}=-0.335)$ and albumin $(\mathrm{B}=-0.289)$.

The excluded variables were hemoglobin, TLC, urea, creatinine, phosphorus, sodium, potassium, vit D as they were not found significant.

Findings: Pre- test score was $(9.766 \pm 1.887)$ and the post test score of sleep quality was $(7.60 \pm 2.175)$.

$\mathrm{P}<0.001$ "There is significant effect of exercise on sleep quality in patients with End Stage Renal Disease on Maintenance Hemodialysis". 
There is decrease in percentage of fairly bad sleep lie from $90 \%$ to $53.3 \%$ and increase in percentage of fairly good sleep lie from $6.7 \%$ to $43.3 \%$.

All the components were significant because there $\mathrm{p}$ value was $<0.005$ and daytime functions was not significant as its $p$ value was $>0.05$.

As sleep medication was our exclusion criteria, no patient were choosen who are on sleep medication.

A stepwise regression test states that On the basis of standardized B coefficients the order of influence on sleep quality was calcium $(B=0.496)$, followed by platelets $(B=0.457)$, chloride $(B=-0.335)$ and albumin $(B=-0.289)$.

The excluded variables were hemoglobin, TLC, urea, creatinine, phosphorus, sodium, potassium, vitamin $\mathrm{D}$ as they were not found significant.

\section{Conclusion}

The total number of sample was $3090 \%$ of patients were having fairly bad sleep quality before the intervention. The pretest score is $(9.7667+1.887)$ and the post test score of the study is $(7.600 \pm 2.175)$. The $p$ value of study is .001; this showed that there is positive effect of the exercise regimen on end stage renal disease on maintenance haemodialysis. According to standardized $\mathrm{B}$ coefficients these four variables influencing the sleep quality of the patients and the $\mathrm{B}$ coefficient is calcium (B $=0.496)$, followed by platelets $(\mathrm{B}=0.457)$, chloride $(\mathrm{B}$ $=-0.335)$ and albumin $(\mathrm{B}=-0.289)$.

This states that only calcium, platelets, chloride and albumin are influencing the QOS of the patients. This means that except these four variables the result of good sleep quality is occurred due to the exercise

The researcher concluded from the bstudy that there is a positive effect of an exercise on quality of sleep of patients which were on maintenance hemodialysis.

Conflict of Interest: There was no conflict of interest in the statement.

Source of Funding: Nil
Ethical Clearance: Ethical clearance taken from the Dialysis unit of the Fortis Hospital, Mohali, Punjab.

\section{References}

1. Merlino G, Piani A, Dolso P, Adorati M, Cancelli I, Valente M, Gigli GL. Sleep disorders in patients with end-stage renal disease undergoing dialysis therapy. Nephrology Dialysis Transplantation. 2006 Jan 1;21(1):184-90.

2. Maung SC, El Sara A, Chapman C, Cohen D, Cukor D. Sleep disorders and chronic kidney disease. World journal of nephrology. 2016 May 6;5(3):224 .

3. Spiegel K, Leproult R, L'Hermite-Balériaux M, Copinschi G, Penev PD, Van Cauter E. Leptin levels are dependent on sleep duration: relationships with sympathovagal balance, carbohydrate regulation, cortisol and thyrotropin. The Journal of clinical endocrinology \& metabolism. 2004 Nov 1;89(11):5762-71.

4. Hilderth C. Prognostic indicators of cardiovascular risk in renal disease. Frontiers in Physiology. 2012 Jan 12;2:121.

5. Karasek M, Szuflet A, Chrzanowski W, Zylinska K, Swietoslawski J. Decreased melatonin nocturnal concentrations in hemodialyzed patients. Neuroendocrinology Letters. 2005 Dec 1;26(6):653-6

6. Elias RM, Bradley TD, Kasai T,Motwani SS,Chan CT. Rostral overnight fluid shift in end stage renal disease: relationship with obstructive sleep apnea. Nephrology Dialysis Transplantation. 2012 April 1;27(4): 1569-73.

7. Science \& your lifestyle, The science of sleep : How exercise impact sleep quality ? National Sleep Foundation [sleep foundation.org]. 2014 [cited 2018 april 24]. Available from :https:// www.sleepfoundation.org/articles/how-exerciseimpacts-sleep-quality.

8. Buysse DJ, Reynolds CF, Monk TH, Berman SR, Kupfer DJ. The Pittsburgh Sleep Quality Index: new instrument for psychiatric practice and research. Psychiatry Res 1989;28: 193-213. 